\title{
Plasma temperature rise toward the plasma-facing surface
}

\author{
D. Nishijima ${ }^{\mathrm{a} *}$, R.P. Doerner ${ }^{\mathrm{a}}$, R.P. Seraydarian ${ }^{\mathrm{a}}$, G. De Temmerman ${ }^{\mathrm{b}}$, and H.J. van der Meiden ${ }^{\mathrm{b}}$ \\ ${ }^{a}$ Center for Energy Research, University of California at San Diego, \\ 9500 Gilman Dr., La Jolla, CA 92093-0417, USA \\ ${ }^{b}$ FOM Institute DIFFER, Dutch Institute For Fundamental Energy Research, Trilateral Euregio Cluster, \\ Nieuwegein, The Netherlands
}

\begin{abstract}
Detailed measurements of axial electron temperature, $T_{\mathrm{e}}$, profiles in the presheath region were carried out using a Langmuir probe and the line intensity ratio technique for both He I (728.1 $\mathrm{nm} / 706.5 \mathrm{~nm})$ and Be II $(467.3 \mathrm{~nm} / 313.1 \mathrm{~nm})$. The results show that $T_{\mathrm{e}}$ increases toward the material surface, which contradicts the standard picture that $T_{\mathrm{e}}$ is constant along the magnetic field in the sheath-limited regime. While no target bias voltage, $V_{\mathrm{b}}$, dependence is seen, the $T_{\mathrm{e}}$ rise becomes more prominent with decreasing neutral pressure. Similarly, the ion temperature, $T_{\mathrm{i}}$, evaluated from Doppler broadening of a He II line emission at $468.6 \mathrm{~nm}$ is found to increase toward the surface, but also does not depend on $V_{\mathrm{b}}$. Possible mechanisms of the $T_{\mathrm{e}}$ and $T_{\mathrm{i}}$ rise as well as validity of the line intensity ratio technique near the material surface are discussed.
\end{abstract}

PACS: 52.40.Hf, 52.40.Kh

PSI-21 Keywords: Presheath, Langmuir probe, Optical spectroscopy 
*Corresponding author address: Center for Energy Research, University of California at San

Diego, 9500 Gilman Dr. Mail Code: 0417, La Jolla, CA 92093, USA

*Corresponding author e-mail: dnishijima@eng.ucsd.edu

Presenting author: Daisuke Nishijima

Presenting author e-mail: dnishijima@eng.ucsd.edu 


\section{Introduction}

The electron temperature, $T_{\mathrm{e}}$, is an essential parameter for understanding both plasma-gas and plasma-material interactions. Electron-impact collision processes depend strongly on $T_{\mathrm{e}}$ [1], and play an important role in the formation of detached plasmas [2]. The photon emissivity coefficient, PEC, and the ionization events per photon, $S / X B$, are also a function of $T_{\mathrm{e}}$ [3]. These parameters are critical to obtain particle densities and sputtered impurity fluxes from line emission intensities. The sputtering yield of material is an important factor in determining the lifetime of plasma-facing components, and is a strong function of the incident ion energy [4], which is mainly determined by $T_{\mathrm{e}}$ via the sheath potential drop. The heat load onto the surface is also affected by $T_{\mathrm{e}}$ through the sheath energy transmission factor [5]. It should be noted that both the sheath potential drop and the sheath energy transmission factor are also affected by the ion temperature, $T_{\mathrm{i}}$.

It is normally assumed that $T_{\mathrm{e}}$ is constant along the magnetic field in the presheath region toward a material surface in the sheath-limited regime [5]. On the other hand, it was reported from the PISCES-A linear device that $T_{\mathrm{e}}$ inferred from the difference between the space and floating potentials becomes higher near the material surface in a plasma containing a significant amount $(10 \%)$ of hot electrons $\left(T_{\mathrm{e}}^{\mathrm{h}} \sim 30 \mathrm{eV}\right.$, compared to the cold bulk $\left.T_{\mathrm{e}}^{\mathrm{c}} \sim 12 \mathrm{eV}\right)$ [6]. It was claimed that the existence of hot electrons is essential for the effective $T_{\mathrm{e}}$ rise. In linear devices like PISCES, the hot electron component originates from primary electrons from the discharge plasma source. In the boundary plasma of tokamaks, hot electrons can also exist, and were confirmed with Langmuir probe measurements in the CASTOR tokamak [7]. 
In this paper, we experimentally investigate axial $T_{\mathrm{e}}$ profiles near the target in plasmas, where the hot electron component is negligibly small $(\leq 0.1 \%$, if any). Both spectroscopic and Langmuir probe methods are used to measure $T_{\mathrm{e}}$. In addition, the $\mathrm{He}^{+}$ion temperature, $T_{\mathrm{He}}$, and the neutral $\mathrm{He}$ temperature, $T_{\mathrm{He}}$, are evaluated from Doppler broadening of spectral lines.

\section{Experimental setup}

The experiments were performed in the PISCES-B linear device [8]. In Fig. 1, the target region is schematically shown. Two spectroscopic systems were used, i.e. one for $T_{\mathrm{e}}$ and another for $T_{\mathrm{He}^{+}}$ and $T_{\mathrm{He}}$. The first system consists of a $0.5 \mathrm{~m}$ Czerny-Turner type spectrometer (Acton SP2560) equipped with a 2-D CCD camera (Princeton Instruments PIXIS:400B). Plasma light emission is directly transferred to the entrance slit of the spectrometer via a plane mirror, a Dove prism, and a focusing lens. Since the image is rotated with the Dove prism, the axial profile is collected in a single acquisition. The spatial resolution is $1.34 \mathrm{~mm}$ per CCD channel. This system measures axial profiles of line intensity ratios of He I $(728.1 \mathrm{~nm} / 706.5 \mathrm{~nm})$ [9] and Be II $(467.3 \mathrm{~nm} / 313.1$ $\mathrm{nm}$ ) [10], which are sensitive to $T_{\mathrm{e}}$. The line-of-sight (LOS) of this system is indicated with a dashed line in Fig. 1.

The second system is employed to measure $T_{\mathrm{He}+}$ and $T_{\mathrm{He}}$ from Doppler broadening of a He II line at $468.6 \mathrm{~nm}$ and $\mathrm{He}$ I line at $492.2 \mathrm{~nm}$, respectively. Plasma emission is delivered via an optical fiber to the entrance slit of a $1.33 \mathrm{~m}$ Czerny-Turner type spectrometer (McPherson 209 ) with a 2-D ICCD camera (Princeton Instruments). The line shape is measured in second

order for better spectral resolution [9]. The dispersion of the system is $5.95 \times 10^{-3} \mathrm{~nm} / \mathrm{pixel}$ at 
$468.6 \mathrm{~nm}$ and $5.78 \times 10^{-3} \mathrm{~nm} /$ pixel at $492.2 \mathrm{~nm}$. Since the instrumental function is Lorentzian with a full width at half maximum (FWHM) of $\sim 8 \times 10^{-3} \mathrm{~nm}$, the measured line is fitted with a Voigt function. For the He II line, 13 fine structures are taken into account for the fit. It should be noted that the FWHM of Stark broadening of the He II line at a typical electron density $n_{\mathrm{e}}=10^{19} \mathrm{~m}^{-3}$ in our experiment presented here is calculated to be less than $2.5 \times 10^{-4} \mathrm{~nm}$ [11], which is negligibly small compared to that of Doppler broadening $\left(\sim 1.3 \times 10^{-2} \mathrm{~nm}\right.$ at $\left.T_{\mathrm{He}+}=0.5 \mathrm{eV}\right)$. The LOS of this system (the diameter of the spot $\sim 10 \mathrm{~mm}$ ) is fixed, and is the same axial location as the reciprocating single probe system. Thus, the target position, defined as $z=0 \mathrm{~mm}$, is axially scanned to obtain axial profiles.

Fig. 2 presents examples of measured electron current $\left(I_{\mathrm{e}}\right)$-applied probe voltage $\left(V_{\mathrm{p}}\right)$ characteristics of the single probe measured at $z=5 \mathrm{~mm}$ in He plasmas at different neutral pressures $P_{\mathrm{He}}=8$ and 2 mTorr. As can be seen, the measured data is well fitted with a single Maxwellian up to $V_{\mathrm{p}}=-80 \mathrm{~V}$ even at $P_{\mathrm{He}}=2 \mathrm{mTorr}$. Calculated bi-Maxwellian curves are also shown, where the fraction of hot electrons $\beta_{\mathrm{h}}=0.1 \%$ and $1 \%$, and the temperature $T_{\mathrm{e}}^{\mathrm{h}}=30,50$, and $100 \mathrm{eV}$ are assumed. $\beta_{\mathrm{h}}$ is defined as $n_{\mathrm{e}}{ }^{\mathrm{h}} /\left(n_{\mathrm{e}}^{\mathrm{c}}+n_{\mathrm{e}}{ }^{\mathrm{h}}\right)$ with the bulk cold electron density, $n_{\mathrm{e}}{ }^{\mathrm{c}}$, and the hot electron density, $n_{\mathrm{e}}^{\mathrm{h}}$. From comparison with the calculated bi-Maxwellian curves, $\beta_{\mathrm{h}}$ is expected to be of the order of $0.1 \%$ or below, which is much smaller than that in Ref. [6].

\section{Experimental results}

\subsection{Neutral pressure scan}

The $P_{\mathrm{He}}$ dependence of axial $T_{\mathrm{e}}$ profiles measured with the single probe is displayed with 
symbols in Fig. 3 (a). Each plotted point is the average of $\sim 5-10$ data points collected around the center $(r \leq 10 \mathrm{~mm})$ of the plasma column from 3 probe shots. The radial profiles of $T_{\mathrm{e}}$ as well as $n_{\mathrm{e}}$ are nearly flat at $r \leq 10 \mathrm{~mm}$ [12]. Error bars are the standard deviation of the mean. In this example, Be was seeded with a high-temperature effusion cell into He plasma, which was terminated with a Mo target at the floating potential. With decreasing $P_{\mathrm{He}}$, the $T_{\mathrm{e}}$ rise toward the target is found to become more prominent. Since $T_{\mathrm{e}}$ is proportional to the difference between the space, $V_{\mathrm{s}}$, and floating, $V_{\mathrm{f}}$, potentials [5], $\left(V_{\mathrm{s}}-V_{\mathrm{f}}\right) / 3.5$ is also shown in Fig. 3 (a), the trend of which is consistent with that of $T_{\mathrm{e}}$.

Measured line intensity ratios of both He I and Be II, presented in Fig. 3 (b) and (c), also indicate the $T_{\mathrm{e}}$ rise, because the ratios increase with an increase in $T_{\mathrm{e}}$, as shown in Fig. 3 (d). The He I and Be II ratios are calculated with the Goto code [13] and the ADAS database [14], respectively. Since $T_{\mathrm{He}}$ was not measured, the radiation trapping effect cannot be precisely calculated [9], and $T_{\mathrm{e}}$ is not evaluated from the He I line intensity ratio here. Inferred $T_{\mathrm{e}}$ from the Be II line intensity ratio is plotted with solid lines in Fig. 3 (a). The line intensity ratio technique seems to be more sensitive to $T_{\mathrm{e}}$ than the single probe, and the $T_{\mathrm{e}}$ rise is visible even at higher $P_{\mathrm{He}}$.

\subsection{Target bias voltage scan}

In Fig. 4 (a), axial $T_{\mathrm{e}}$ profiles (top), obtained with the He I line intensity ratios (bottom), are plotted as a function of the target bias voltage, $V_{\mathrm{b}}$, which determines the incident ion energy to the target as $E_{\mathrm{i}}=V_{\mathrm{s}}-V_{\mathrm{b}}$. In this experiment, $V_{\mathrm{s}}$ was around $-4 \mathrm{~V}$ in the upstream, and a W sample 
was exposed to pure He plasma with $P_{\mathrm{He}} \sim 5$ mTorr. It can be seen that the axial $T_{\mathrm{e}}$ profile does not depend on $V_{\mathrm{b}}$. This is consistent with probe measurements, as shown with symbols.

Axial $T_{\mathrm{He}+}$ and $T_{\mathrm{He}}$ profiles are shown in Fig. 4 (b) and (c), respectively. Since (1) the discharge power mainly goes to electrons and (2) ions can effectively transfer the energy to neutrals through charge-exchange and elastic collisions, $T_{\mathrm{He}}$ is typically much lower than $T_{\mathrm{e}}$ in linear devices. Furthermore, $T_{\mathrm{He}}$ is also found to increase toward the target, while the $T_{\mathrm{He}}$ profile is flat. Similarly to $T_{\mathrm{e}}$, both $T_{\mathrm{He}+}$ and $T_{\mathrm{He}}$ show no $V_{\mathrm{b}}$ dependence. It is worth noting that the $T_{\mathrm{He}+}$ rise seems to start at $z \sim 30 \mathrm{~mm}$, while $T_{\mathrm{e}}$ starts to increase at $z \sim 10 \mathrm{~mm}$. This may imply that the heating mechanism is different between electrons and ions.

\section{Discussion}

\subsection{Validity of the line intensity ratio technique near the material surface}

It is known that energetic ion bombardment on material surfaces can lead to the formation of excited states of reflected projectiles and sputtered particles [15]. Most of reported experiments were conducted at much higher $E_{\mathrm{i}}$ above $\mathrm{keV}$ with ion beams compared to the present experiments $(\leq 100 \mathrm{eV})$. Although the excitation rate generally decreases with decreasing $E_{\mathrm{i}}$, we cannot completely exclude a possibility that the He I line intensity ratio is affected by reflected neutrals in excited states. On the other hand, it is considered that $\mathrm{Be}^{+}$ions are produced purely in the plasma. Although a fraction of sputtered species from the surface is generally ions, ions cannot overcome the sheath potential in front of the material surface in the plasma environment. Thus, the Be II line intensity ratio is not influenced by excited state ions formed during 
sputtering.

The Be II line at $313.1 \mathrm{~nm}$ is the principal resonance transition, and it can be reabsorbed when the $\mathrm{Be}^{+}$ion density, $n_{\mathrm{Be}^{+}}$, is high enough. The reabsorption results in a reduction of the emission intensity and, therefore, in an increase in the intensity ratio $467.3 \mathrm{~nm} / 313.1 \mathrm{~nm}$. To see if reabsorption occurs in our plasmas, the ratio was measured away from the target in plasmas at $T_{\mathrm{e}} \sim 5.5 \mathrm{eV}$, while $n_{\mathrm{Be}+}$ was scanned by changing the Be seeding rate. As demonstrated in Fig. 5, the measured ratio is constant up to the highest achieved $n_{\mathrm{Be}+} \sim 0.3 \times 10^{16} \mathrm{~m}^{-3}$, which is much higher than the range in Fig. 3 (c). In fact, the optical depth, $\tau$, calculated with the formula in [16], is below $10^{-2}$ even at $n_{\mathrm{Be}^{+}} \sim 0.3 \times 10^{16} \mathrm{~m}^{-3}$ as seen in Fig. 5, meaning that our plasmas are optically thin for the Be II $313.1 \mathrm{~nm}$ line. Thus, the Be II line intensity ratio is not affected by reabsorption.

There are two metastable states $\left(2{ }^{1} \mathrm{~S}\right.$ and $\left.2^{3} \mathrm{~S}\right)$ in the He I system, which have a long radiative lifetime. There is, therefore, a possibility that metastable atoms produced in the upstream may flow toward the target, and may affect the population distribution near the target. However, metastable atoms can travel less than $1 \mathrm{~mm}$ before being excited to another state due to electron impact, estimated using $n_{\mathrm{e}}=10^{19} \mathrm{~m}^{-3}, T_{\mathrm{He}}=0.12 \mathrm{eV}$, and the rate coefficient for electron impact excitation $\langle\sigma v\rangle_{\text {en }} \sim 10^{-12} \mathrm{~m}^{3} / \mathrm{s}$ [1]. Thus, the transport of metastable He atoms is not important in our conditions. Note that, in the Be II system, there is no metastable state.

As can be seen in Fig. 3 (d), the Be II line intensity ratio increases with an increase in $n_{\mathrm{e}}$. The He I line intensity ratio also increases with $n_{\mathrm{e}}$ at $n_{\mathrm{e}} \sim 10^{19} \mathrm{~m}^{-3}$ [9], although the dependency is very weak. However, probe measurements showed that $n_{\mathrm{e}}$ monotonically goes 
down toward the target, as expected in the sheath-limited regime. Thus, the observed increase in both line intensity ratios toward the target cannot be explained by a change in $n_{\mathrm{e}}$. This also confirms that the effect of recycling neutrals is small.

\subsection{Possible mechanisms for the plasma temperature rise}

First, high energy reflected neutrals originating from ions accelerated in the sheath may heat up ions in the plasma. However, the mean free path, $\lambda_{\mathrm{mfp}}$, of reflected He atoms for charge exchange with $\mathrm{He}^{+}$ions is calculated to be more than $0.5 \mathrm{~m}$ in our conditions, which is much longer than the observed characteristic length $(\sim 30 \mathrm{~mm})$ of the $T_{\mathrm{i}}$ rise. Here, the $\mathrm{He}^{+}$ion density is $\sim 10^{19} \mathrm{~m}^{-3}$, and the cross section is $\sim 1.5-2 \times 10^{-19} \mathrm{~m}^{2}$ [1]. The $\lambda_{\mathrm{mfp}}$ for elastic collisions is expected to be similar to that for charge exchange.

Secondary electrons emitted from the surface gain energy in the sheath, and may couple energy to the plasma through collisions. The electron-ion collision time is written as,

$$
e(\mathrm{~s})=3.4410^{11} \frac{T_{e}^{1.5}}{n_{i} \ln },
$$

with $T_{\mathrm{e}}$ in $\mathrm{eV}$ and $n_{\mathrm{i}}$ in $\mathrm{m}^{-3}$ for singly charged ions [17]. With $T_{\mathrm{e}}=20-100 \mathrm{eV}$ for secondary electrons accelerated in the sheath, the ion density $n_{\mathrm{He}+}=n_{\mathrm{e}}=10^{19} \mathrm{~m}^{-3}$, and the Coulomb logarithm $\ln \Lambda=10, \tau_{\mathrm{e}}$ is calculated to be $\sim 0.3-3 \times 10^{-6} \mathrm{~s}$. Then, the characteristic length becomes $\sim 0.8-20 \mathrm{~m}\left(\gg>30 \mathrm{~mm}\right.$ for the $T_{\mathrm{i}}$ rise $)$ with the electron velocity $v_{\mathrm{e}}=\left(2 e T_{\mathrm{e}} / m_{\mathrm{e}}\right)^{0.5}$. Here, $e$ is the elementary charge. The electron-electron collision time and energy exchange time are even longer. As a consequence, the effect of secondary electrons on the local heating near the surface can be also excluded. 
Non-Maxwellian electron effects were proposed by LaBombard et al [6]. To describe the $n_{\mathrm{e}}$ profile in the presheath with two $T_{\mathrm{e}}$ components, a modified Boltzmann law is written as,

$$
n_{e}(z)=n_{e 0}^{c} \exp \frac{V_{s}(z) V_{s 0}}{T_{e}^{c}}+n_{e 0}^{h} \exp \frac{V_{s}(z) V_{s 0}}{T_{e}^{h}},
$$

where the subscript 0 indicates the quantity in the upstream, and the superscript $\mathrm{c}$ and $\mathrm{h}$ mean cold (bulk) and hot electron components. A presheath potential drop of $\sim 0.5 \times T_{\mathrm{e}}^{\mathrm{c}}$ leads to a decrease in the bulk electron component, i.e. $\sim 0.6 \mathrm{x} n_{\mathrm{e} 0}{ }^{\mathrm{c}}$, while the hot component is relatively unaffected. Thus, the effective $T_{\mathrm{e}}$ near the surface can become higher than the upstream one. In our plasmas, the fraction of hot electrons is of the order of $0.1 \%$ or below, much smaller than that in [6]. However, the fraction and energy of hot electrons are expected to increase with increasing the discharge voltage, $V_{\mathrm{d}}$, as $P_{\mathrm{He}}$ decreases [Fig. 3 (e)]. (Note that the $V_{\mathrm{d}}$ increase is spontaneously induced to sustain the plasma discharge.) This is because hot electrons originate from primary electrons from the discharge cathode, and a higher $V_{\mathrm{d}}$ leads to a higher energy of primary electrons. With decreasing $P_{\mathrm{He}}, n_{\mathrm{e}}$ decreases, and thus primary electrons are less thermalized because of fewer collisions with the background plasma and neutral gas, resulting in a higher fraction and energy of hot electrons. As a consequence, the effect of hot electrons cannot be ruled out from candidate mechanisms for the $T_{\mathrm{e}}$ rise. However, this mechanism cannot explain the $T_{\mathrm{i}}$ rise.

In Magnum-PSI [18], axial $T_{\mathrm{e}}$ profiles $(z=3-50 \mathrm{~mm})$ were measured with a Thomson scattering system while scanning the axial position of a $\mathrm{Cu}$ target. By controlling the discharge parameters including the discharge current, the magnetic field strength, and the gas flow rate, $T_{\mathrm{e}}$ and $n_{\mathrm{e}}$ were varied. At $n_{\mathrm{e}} \geq 10^{20} \mathrm{~m}^{-3}, T_{\mathrm{e}}$ is found to go down toward the target, because the plasma 
is not in the sheath-limited regime but in the high-recycling or recombining condition unlike the condition in PISCES-B. At lower $n_{\mathrm{e}}$, the signal to noise ratio near the target was too low to judge the $T_{\mathrm{e}}$ profiles with confidence.

\section{Summary and conclusions}

More energetic plasmas in front of the material surface, when compared to the upstream plasma, have been experimentally observed in the sheath-limited regime with spectroscopic and Langmuir probe methods. Observations of the $T_{\mathrm{e}}$ rise may be consistent with the non-Maxwellian electron model, although the fraction of hot electrons in our plasmas is very small, i.e. $\sim 0.1 \%$ or below. In other words, even a small amount of hot electrons can lead to a higher $T_{\mathrm{e}}$ in front of the material surface. From the simple estimate of the characteristic lengths, the effects of high-energy reflected neutrals and secondary electron emission can be excluded from the candidate for the $T_{\mathrm{e}}$ and $T_{\mathrm{i}}$ rise. However, more detailed simulations would be necessary to verify these effects as well as the non-Maxwellian electron model, to find out any other mechanisms, and to clarify the energy balance near the material surface.

\section{Acknowledgements}

We would like to thank technical staff at the PISCES and Magnum-PSI groups for their excellent work. This work is conducted under the US DOE Contract: DE-FG02-07ER54912 and under the US-EU bilateral collaboration. 


\section{References}

[1] R.K. Janev, W.D. Langer, K. Evans Jr., D.E. Post Jr., Elementary Processes in Hydrogen-Helium Plasmas, Springer-Verlag, Berlin Heidelberg, 1987.

[2] S. Takamura et al., Plasma Sources Sci. Technol. 11 (2002) A42.

[3] A. Pospieszczyk et al., J. Phys. B 43 (2010) 144017.

[4] W. Eckstein, Calculated Sputtering, Reflection and Range Values, Report of the Max-Planck-Institute für Plasmaphysik, IPP-Report 9/132, Garching, Germany, 2002.

[5] P.C. Stangeby, The Plasma Boundary of Magnetic Fusion Devices, Institute of Physics Publishing, Bristol and Philadelphia, 2000.

[6] B. LaBombard et al., J. Nucl. Mater. 162-164 (1989) 314.

[7] T.K. Popov et al., Plasma Phys. Control. Fusion 51 (2009) 065014.

[8] R.P. Doerner et al., Physica Scripta T111 (2004) 75.

[9] D. Nishijima and E.M. Hollmann, Plasma Phys. Control. Fusion 49 (2007) 791.

[10] D. Nishijima et al., J. Nucl. Mater. 438 (2013) S1245.

[11] H.R. Griem, Spectral Line Broadening by Plasmas, Academic Press, New York and London, 1974.

[12] D.G. Whyte et al., Nucl. Fusion 41 (2001) 47.

[13] M. Goto, J. Quant. Spectrosc. Radiat. Transfer 76 (2003) 331.

[14] H.P. Summers, The ADAS User Manual version 2.6 (2004).

[15] E.W. Thomas, Progress in Surface Science 10 (1980) 383.

[16] J.D. Huba, NRL Plasma Formulary, The Office of Naval Research, Washington, DC, 1994. 
[17] J. Wesson, Tokamaks 2nd edition, Clarendon Press, Oxford, 1997.

[18] G. De Temmerman et al., Fus. Eng. Des. 88 (2013) 483. 


\section{Figure captions}

Figure 1. Schematic view of the PISCES-B target region. The target surface is defined as $z=0$ $\mathrm{mm}$. The line-of-sight (LOS) of two spectroscopic systems is indicated. The axial position of the reciprocating probe system is the same as the LOS of the high-resolution spectroscopic system for $T_{\mathrm{He}+}$ and $T_{\mathrm{He}}$ measurements.

Figure 2. Measured $I_{\mathrm{e}}-V_{\mathrm{p}}$ characteristics of the single probe in He plasmas at (a) $P_{\mathrm{He}}=8 \mathrm{mTorr}$ and (b) 2 mTorr. The measured data is well fitted with a single Maxwellian. Calculated bi-Maxwellian curves $\left(\beta_{\mathrm{h}}=0.1 \%\right.$ and $1 \%, T_{\mathrm{e}}^{\mathrm{h}}=30,50$, and $\left.100 \mathrm{eV}\right)$ are shown with dashed lines.

Figure 3. $P_{\mathrm{He}}$ scan. Axial profiles of (a) $T_{\mathrm{e}}$ measured with the single probe (symbols), (b) the $T_{\mathrm{e}}$-sensitive He I line intensity ratio: $728.1 \mathrm{~nm} / 706.5 \mathrm{~nm}$, and (c) the $T_{\mathrm{e}}$-sensitive Be II line intensity ratio: $467.3 \mathrm{~nm} / 313.1 \mathrm{~nm}$. In (a), $\left(V_{\mathrm{s}}-V_{\mathrm{f}}\right) / 3.5$ and inferred $T_{\mathrm{e}}$ from the Be II line intensity ratio are also drawn with dashed and solid lines, respectively. Measurements were done in Be-seeded He plasmas with a Mo target at the floating potential. (d) Calculated He I (728.1 $\mathrm{nm} / 706.5 \mathrm{~nm})$ [13] and Be II $(467.3 \mathrm{~nm} / 313.1 \mathrm{~nm})$ [14] ratios. For He I, the effect of radiation trapping (R.T.) is presented [9], while the $n_{\mathrm{e}}$ dependence is shown for Be II. (e) $P_{\mathrm{He}}$ dependence of the discharge voltage $V_{\mathrm{d}}$ and current $I_{\mathrm{d}}$.

Figure 4. $V_{\mathrm{b}}$ scan. Axial profiles of (a) $T_{\mathrm{e}}$ (top) and the He I line intensity ratio: $728.1 \mathrm{~nm} / 706.5$ nm (bottom), (b) $T_{\mathrm{He}^{+}}$, and (c) $T_{\mathrm{He}}$. Measurements were done in pure He plasmas $\left(V_{\mathrm{s}} \sim-4 \mathrm{~V}\right.$ at $z=$ 
$75 \mathrm{~mm}$ ) at $P_{\mathrm{He}} \sim 5 \mathrm{mTorr}$. The target was W. In (a), $T_{\mathrm{e}}$ from probe measurements is also shown with symbols for comparison.

Figure 5. Be II line intensity ratio $467.3 \mathrm{~nm} / 313.1 \mathrm{~nm}$ vs. $n_{\mathrm{Be}+}$, measured far from the target at $T_{\mathrm{e}}$ $\sim 5.5 \mathrm{eV}$. Calculated optical depth $\tau$ of the Be II line at $313.1 \mathrm{~nm}$ is drawn (dashed line). The $n_{\mathrm{Be}+}$ range in Fig. 3 (c) is hatched. 


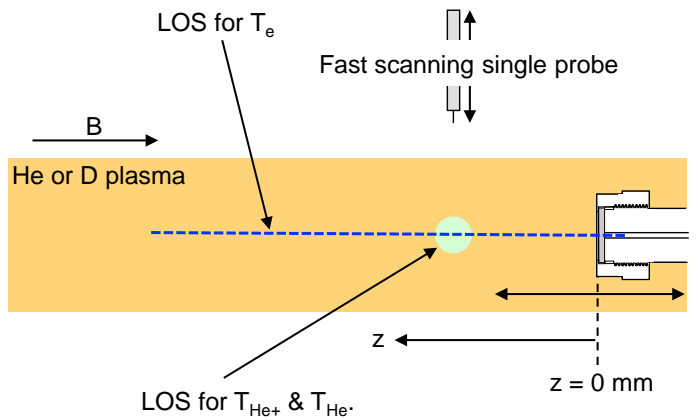

Figure 1. D. Nishijima et al (Single column) 


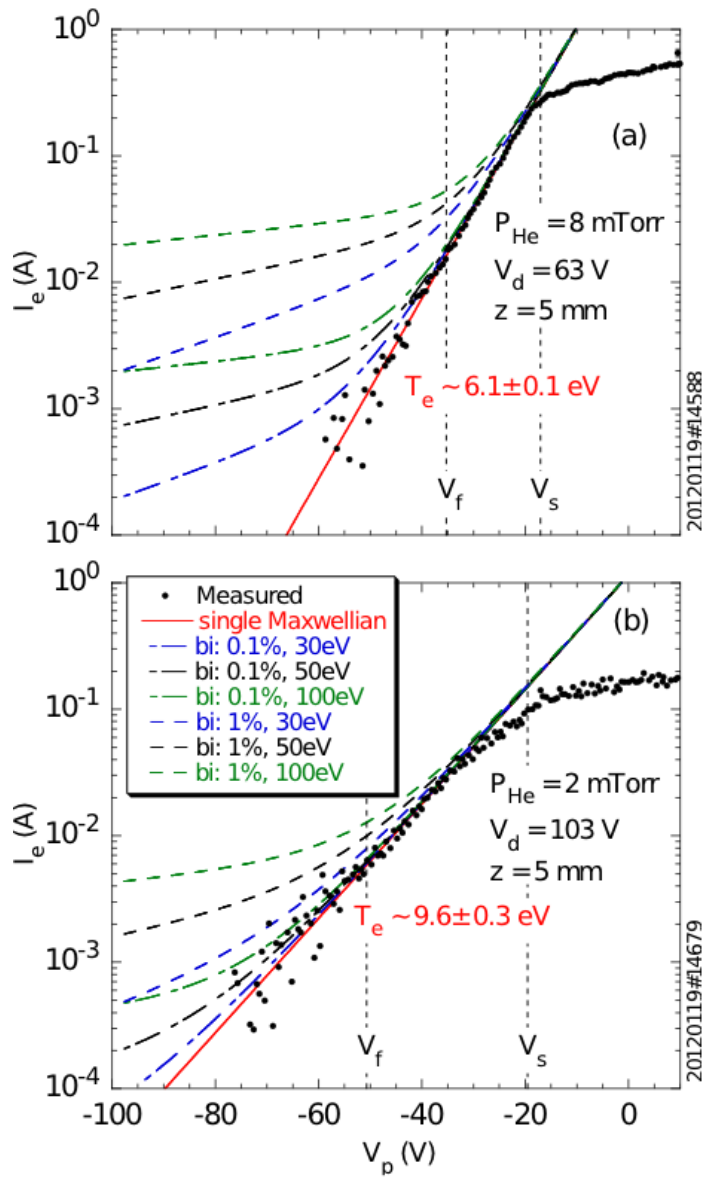

Figure 2. D. Nishijima et al (Single column) 

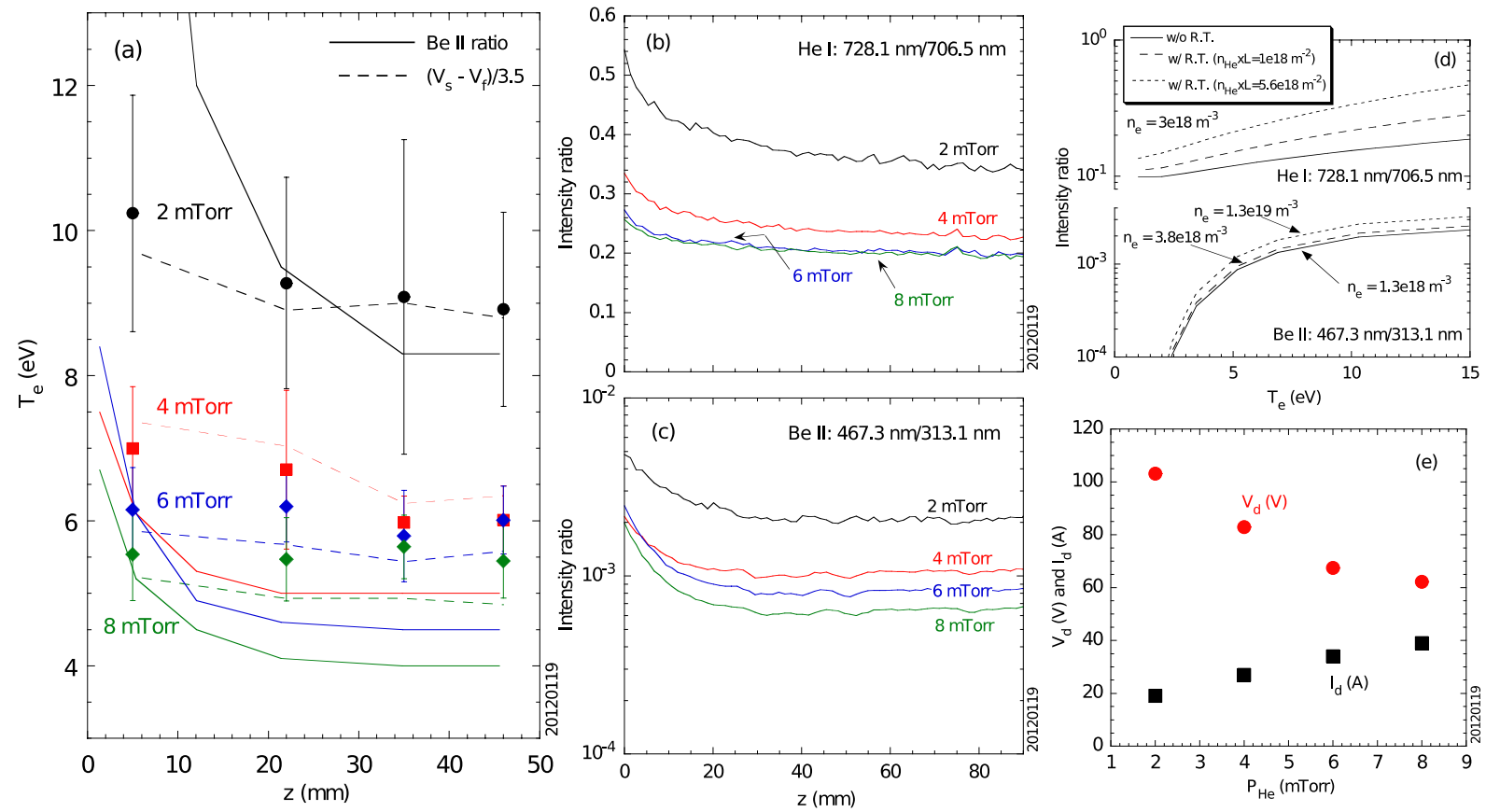

Figure 3. D. Nishijima et al

(Double column) 

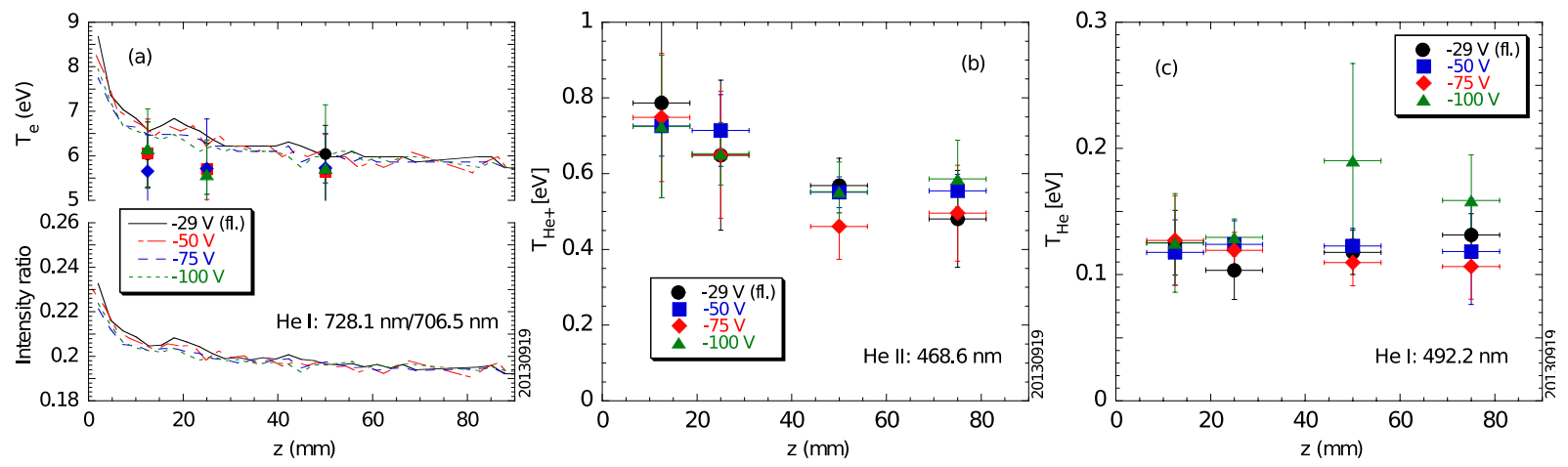

Figure 4. D. Nishijima et al (Double column) 


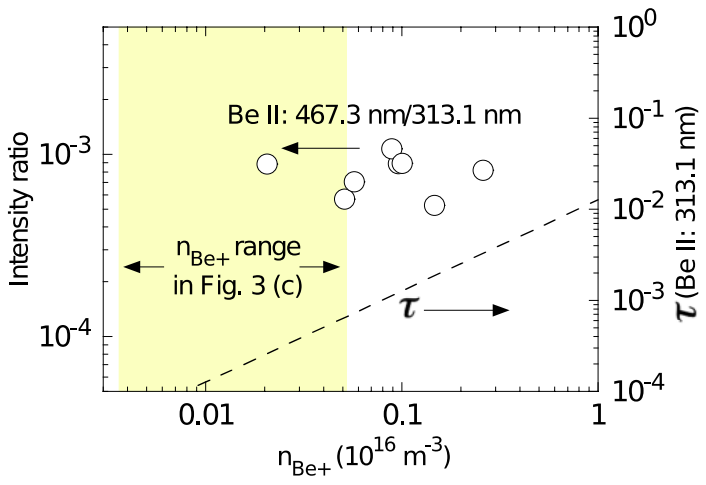

Figure 5. D. Nishijima et al (Single column) 